\title{
Optimizing Student Learning and Retention of Time- and Frequency- Domain Concepts Through Numerical Computation Projects
}

\author{
Steve Warren, Ph.D. \\ Department of Electrical \& Computer Engineering, Kansas State University
}

\begin{abstract}
The ability to analyze signals and systems in both the time and frequency domains is an essential outcome in most engineering curricula. However, concepts such as time-domain convolution and frequency-domain spectra can be elusive to students. While most students can learn the mathematical procedures whereby they obtain correct answers on homework and exams, many lack the higher-level understanding that aids long-term retention. This paper addresses the creation of MATLAB and C projects that facilitate learning in these areas by clearly stating learning objectives, maintaining student interest with real-world applications, partitioning large projects into incremental modules, and providing the repetition necessary for long-term retention. The underlying assumptions for this work are two-fold: (1) if a student can write the numerical software to solve a problem, they understand the theoretical principles well and (2) students receive personal satisfaction and gain confidence when their software produces correct results. The illustrative time-domain projects described here address linear convolution and trigonometric/exponential Fourier series. The frequency-domain projects address the computation of discrete Fourier transforms, spectral assessment, and time-frequency analysis. Real-world applications such as filter cascades for signal conditioning, tone control knobs for audio systems, and parameter extraction from biomedical signals drive these multi-week efforts. Typically assigned in the junior and senior years of the undergraduate curriculum, these efforts are precursors to upper-level undergraduate and graduate projects that incorporate full user interfaces. Through end-of-year evaluations and post-graduate feedback, many students refer to these projects as the highlight of their course experience.
\end{abstract}

\section{Introduction}

Linear systems (a.k.a., signals and systems) courses typically address both time- and frequencydomain principles. Continuous and sampled signals are important in both domains. While linear superposition and signal characteristics (e.g., behavior and symmetry) are conceptually straightforward for students, convolution is an elusive concept. Although it may have already been introduced in a previous circuit theory course, it was most likely presented as a flip-andshift, or fold-and-slide, approach that represents the mathematics but is not physically intuitive. Additionally, while the Fourier series approach to building arbitrary signals with sinusoids makes sense to students, the mathematics involved with calculating the Fourier coefficients can be difficult for the average student. Students may also learn about Laplace transforms and filter transfer functions in an earlier circuit theory course. While they can demonstrate frequencydependent behavior with analog circuits in the laboratory, they find it difficult to (a) conceptually map time-domain signal character to frequency-domain spectra and (b) describe the effect of a frequency-domain filter on the shape of a time-domain signal, even if they understand the 
fundamental concept of a Fourier series. Finally, linear systems students find it hard to correctly interpret the arrays returned by discrete spectral analysis functions, such as canned Fast Fourier Transform (FFT) routines, because of the multiple relationships between the time- and frequency-domain parameters. They find it even more difficult to define discrete frequencydomain filter arrays that must align with the input signal spectra; correct placement of the negative frequency coefficients seems to be especially problematic.

From intuition and experience, instructors understand that if a student can write numerical code to accomplish a convolution, a Fourier series transformation, and a frequency-domain filtering process, then the student understands these concepts at a high-enough level of abstraction ${ }^{1,2}$ that they are likely to retain the material for a greater period of time. Research geared toward computation, hands-on learning, and retention supports this assertion. ${ }^{3-5}$ Computers add a visual and interactive element that resonates with students today. ${ }^{6-8}$ Research efforts confirm what most educators know: creative projects that actively engage students inside and outside of the classroom result in increased learning, a more positive educational experience, and improved teacher/student interactions.

Research and experience indicate that computational projects can address difficult-to-learn subjects in a course like Linear Systems because students (1) generally enjoy interacting with computers, (2) gain satisfaction from creating a working computer code, and (3) must understand the theory in order to obtain correct numerical results. Learning theory is a prerequisite to a functional project. This paper presents a collection of numerical computation projects in the areas of convolution, Fourier series, and Fast Fourier Transforms that have been recently utilized in EECE 512 - Linear Systems, a course offered by the Department of Electrical \& Computer Engineering (EECE) at Kansas State University (KSU). These programming projects supplement handwritten work and represent approximately $15 \%$ of a semester course grade. The following sections address the theory, learning objectives, and administration of these projects while presenting application examples. This is followed by an assessment of the learning effectiveness of these projects.

\section{Theory}

Convolution. If a system's impulse response is known, the zero-state response of the system (response with no initial stored energy) due to an input signal can be determined by performing a convolution between the input signal and the impulse response. ${ }^{9-12}$ The convolution process assumes that the input signal can be approximated by an infinite number of closely spaced samples, where each sample is represented by a weighted impulse that acts at the sample time:

$$
f(t)=\lim _{\Delta \tau \rightarrow 0} \sum_{n=-\infty}^{\infty} f(n \Delta \tau) \delta(t-n \Delta \tau) \Delta \tau,
$$

where $n=0,1, \ldots$ is a sample index and $\Delta \tau$ is the sample spacing. This notation is from Lathi. ${ }^{9}$ The system responds to each impulse in turn, so the output signal is the sum, or superposition, of the responses to the individual impulses that comprise the input signal:

$$
y(t)=\lim _{\Delta \tau \rightarrow 0} \sum_{n=-\infty}^{\infty} f(n \Delta \tau) h(t-n \Delta \tau) \Delta \tau .
$$


This expression is an intuitive representation of the physical process and can be referred to as 'physical' convolution. Most texts move directly to the integral form,

$$
y(t)=\int_{-\infty}^{\infty} f(\tau) h(t-\tau) d \tau=\int_{-\infty}^{\infty} h(\tau) f(t-\tau) d \tau,
$$

which is usually explained by a fold-and-slide (or flip-and-shift) process that graphically explains the mathematics but does not represent an intuitive means to visualize the process.

When physical convolution is implemented by a student on a computer, the calculations can follow the natural behavior of the system, where the system responds to each weighted impulse in turn. Both the input signal and the impulse response are assumed to begin at time zero: the system is causal. The response to an impulse at time zero is first determined, where the impulse is weighted by the value of the function and the sample spacing $\Delta \tau$. Next, the response to a weighted impulse at time $\Delta \tau$ is determined, and this response is added to the response from the impulse at time zero. The output signal now consists of the responses from the first two weighted impulses. The responses from the input impulses at times $2 \Delta \tau, 3 \Delta \tau$, etc. are added to the output signal until it is fully constructed. This convolution process is represented numerically by the following sum:

$$
y[n]=\sum_{i=0}^{n} f[i] h[n-i] \Delta \tau, n=0, \ldots, \infty
$$

where $f(t)$ and $h(t)$ are assumed to exist for all time. The length of the $y[]$ array is actually the sum of the lengths of the $f[]$ and $h[]$ arrays minus 1 . Once the process is understood by a student, they find they can implement physical convolution in MATLAB ${ }^{13,14}$ with very few lines of code:

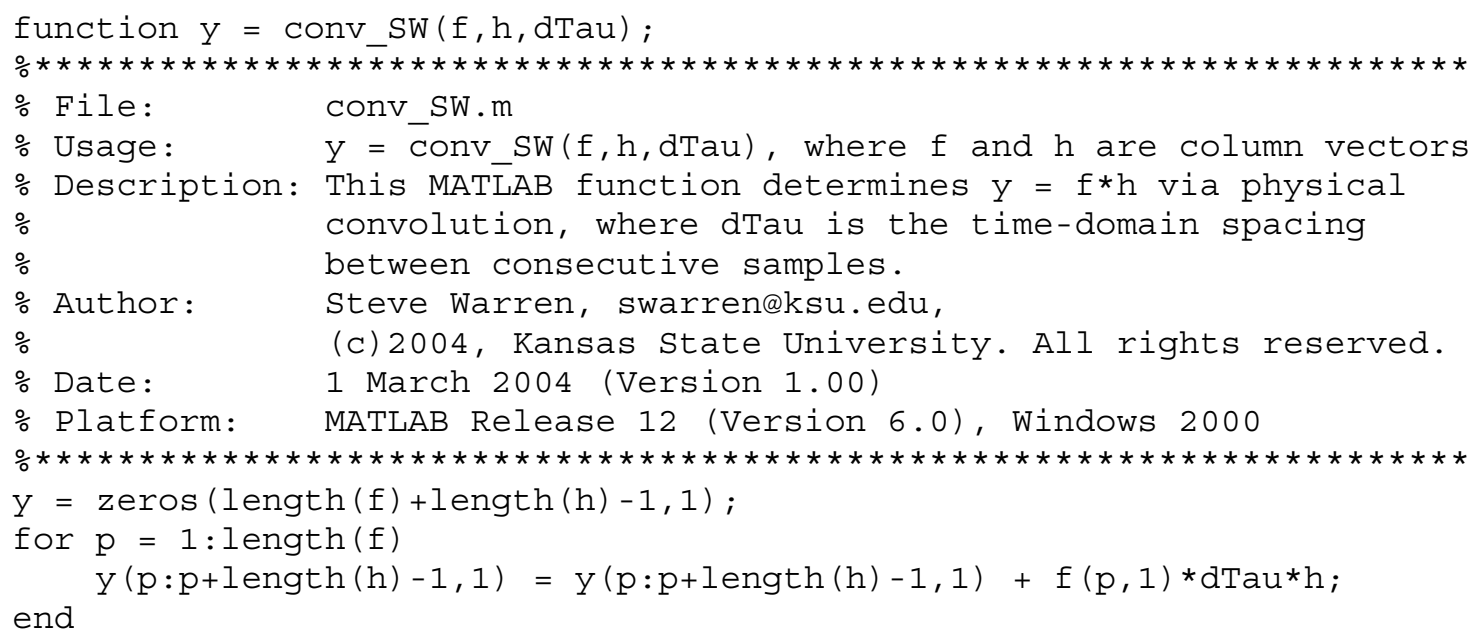

Fourier Series. Any periodic signal, $f(t)$, can be decomposed into a sum of sinusoids, each with a different magnitude, phase, and frequency. This trigonometric Fourier series, $f_{T F S}(t)$, is expressed as

$$
f_{T F S}(t)=a_{0}+\sum_{n=1}^{\infty} a_{n} \cos \left(2 \pi n f_{0} t\right)+b_{n} \sin \left(2 \pi n f_{0} t\right) .
$$

Here, $a_{0}$ is the DC, or average, value of the signal over a given time interval of duration $T_{0}=1 / f_{0}$ seconds $\left(f_{0}=\omega_{0} / 2 \pi\right.$ is referred to as the "fundamental" frequency): 


$$
a_{0}=\frac{1}{T_{0}} \int_{t_{1}}^{t_{1}+T_{0}} f(t) d t
$$

The coefficients $a_{n}$ and $b_{n}$ represent the magnitudes of the cosines (even functions) and sines (odd functions) that constitute the signal. These coefficients are determined using the following expressions

$$
a_{n}=\frac{2}{T_{0}} \int_{t_{1}}^{t_{1}+T_{0}} f(t) \cos \left(n \omega_{0} t\right) d t, n=1,2,3, \ldots
$$

and

$$
b_{n}=\frac{2}{T_{0}} \int_{t_{1}}^{t_{1}+T_{0}} f(t) \sin \left(n \omega_{0} t\right) d t, n=1,2,3, \ldots,
$$

where $n$ is an integer that represents the number of harmonics (in addition to $a_{0}$ ) used to reconstruct the signal. If the original signal, $f(t)$, is not periodic, the Fourier series approximation assumes periodicity outside of the original time range (e.g., for $t<t_{1}$ and $t>t_{1}+T_{0}$ ).

Programming these relationships is relatively straightforward for a student if they can first understand the required order of operations before they start to write code. The integrals for $a_{n}$ and $b_{n}$ can be calculated with one of many quadrature rules. ${ }^{15}$ The MATLAB script below uses the trapezoidal quadrature rule.

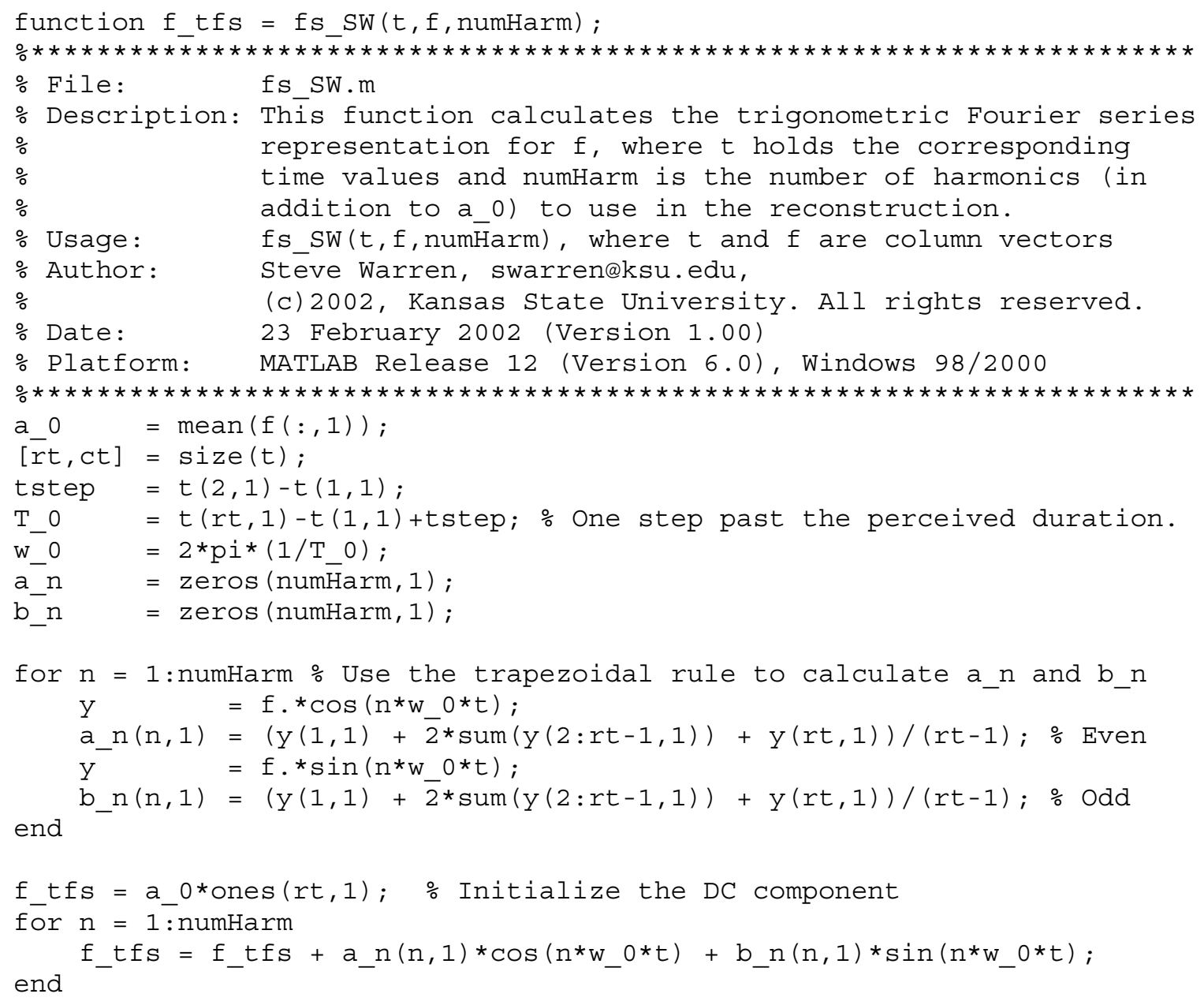

Proceedings of the 2005 American Society for Engineering Education Annual Conference and Exposition Copyright $@ 2005$, American Society for Engineering Education 
Discrete Fourier Transform. The direct Fourier transform of a continuous signal $f(t)$ is

$$
F(\omega)=\int_{-\infty}^{\infty} f(t) e^{-j \omega t} d t
$$

where $F(\omega)$ is complex. The original signal can be reconstructed using

$$
f(t)=\frac{1}{2 \pi} \int_{-\infty}^{\infty} F(\omega) e^{j w t} d \omega .
$$

Numerical computation of the Fourier transform requires that $f(t)$ be sampled at discrete times. Spectral components for this signal can then only be determined for discrete frequencies. The direct discrete Fourier transform (DFT), $F_{r}$, of a sampled signal, $f_{k}$, is given by

$$
F_{r}=\sum_{k=0}^{N_{0}-1} f_{k} e^{-j r \Omega_{0} k}
$$

where $N_{0}$ is the number of points in the sampled sequence, $r=0, \ldots, N_{0^{-}} 1$ is a frequency-domain index, $k=0, \ldots, N_{0^{-}} 1$ is a time-domain index, $\Omega_{0}=\omega_{0} T=2 \pi f_{0} T=2 \pi\left(1 / T_{0}\right) T=2 \pi / N_{0}$ represents an incremental change in angle (in radians), $T_{0}$ is the total sequence duration (plus one sample step) in seconds, $f_{0}$ is the fundamental frequency in $\mathrm{Hz}$, and $T$ is the sample period. In order to retain the spectral information in the original signal, the sample frequency, $f_{s}=1 / T$, should be greater than twice the highest frequency component in the signal. In the discrete domain, the reconstructed signal, $f_{k}$, can be determined using the inverse discrete Fourier transform:

$$
f_{k}=\frac{1}{N_{0}} \sum_{r=0}^{N_{0}-1} F_{r} e^{j r \Omega_{0} k}
$$

An efficient implementation of the direct Fourier transform, called a Fast Fourier Transform (FFT) takes advantages of symmetries and redundancies in the matrix used to calculate the $F_{r}$ coefficients. Undergraduate students will typically apply a canned FFT routine to their signal data and concentrate on (a) properly assigning corresponding frequency values to the returned array and (b) interpreting the resulting magnitude and phase spectra. To verify calculated coefficients with those expected from theory, students may have to apply a scaling factor. For example, coefficients calculated using the Syntopia ${ }^{16}$ fft ( ) routine must be multiplied by $N_{O} / 2 \pi$ in order to match hand calculations using the notation in Lathi. ${ }^{9}$ The $N_{O}$ factor is induced by the FFT algorithm, whereas the $2 \pi$ factor is induced through the conversion from $F(\omega)$ to $F_{r}$ when the independent variable is frequency in $\mathrm{Hz}$.

\section{Methods: Project Descriptions and Sample Products}

Project Approach and Constraints. At KSU, EECE 512: Linear Systems, is taken by juniors and seniors in both the Electrical Engineering and Computer Engineering curricula. When this course is taught with a traditional approach that utilizes in-class lectures and handwritten homework, the amount of material that must be covered in a given semester is significant. However, this course has been chosen by the KSU EECE faculty as a venue where students will receive additional $\mathrm{C}$ programming practice prior to graduation. When programming projects supplement the traditional Linear Systems material, a set of constraints is necessary to make sure that the project learning experience is not overshadowed by the students' dissatisfaction with a real or perceived overcommitment of resources on their part. The constraints applied by the instructor to date include the following:

\footnotetext{
- Each project must have a clear set of learning objectives.
} 
- While C programming is a 'required' project element, MATLAB is also used because of its ability to perform relatively complex operations and plots with fewer lines of code.

- Multi-week problems are preferred because they offer the greatest potential for substantive effort as opposed to small programming efforts that amount to busy work. This places a greater burden on the instructor, who must think through all of the project facets prior to the assignment.

- Staged deadlines help students manage their time. For a month-long project, students may have (a) one week to write the code to load and dynamically store the signal data, (b) a week or two to write the processing code, and (c) another week to create plots and prepare the project submission.

- Projects must be streamlined so that they make optimal use of student time. The general project flow is illustrated in Figure 1. Input data are read from ASCII text files using a console window, avoiding distractions such as graphical user interfaces that can consume significant time. Once the data are processed (see the following sections), the results are sent to output files and then plotted. These program elements ensure that students practice file input/output, learn to write numerical computation code, and understand how to present signal information in a visually informative way. The visual element is important, as students generally need practice equating theoretical expressions with their graphical counterparts. Projects written for MATLAB creates plots directly, while C programming projects produce either a MATLAB or GNUPLOT script that can be called from within the respective programming environment.

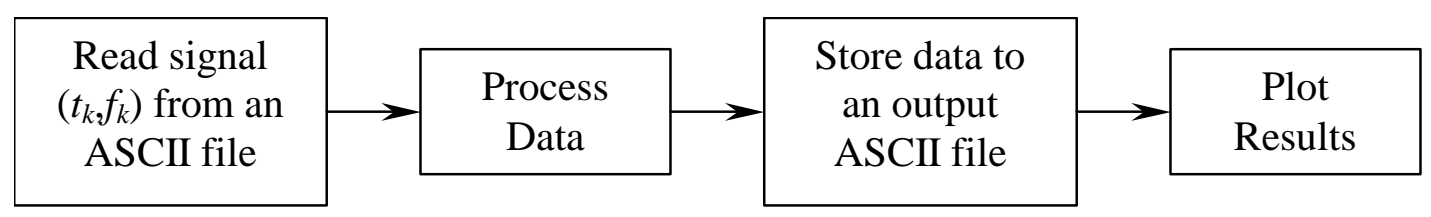

Figure 1. General order of operations for the numerical computation projects.

- When possible, students corroborate calculated results with hand calculations or PSPICE $^{17}$ simulations. This builds confidence and promotes good development habits.

- Projects and their accompanying implementation language (MATLAB or C) rotate from semester to semester to provide variety and discourage students from copying a previous semester's work. A Spring course offering might include a convolution project in $\mathrm{C}$ and a frequency-domain filtering project in MATLAB, whereas a Fall offering might include a Fourier series project in MATLAB and a frequency-domain filtering project in C. From experience, two projects of limited duration per semester is appropriate.

- Microsoft Visual Studio .NET is chosen as the standard application environment within which each submission will be graded. The KSU EECE Department is a member of the Microsoft Academic Alliance,${ }^{18}$ which allows students and faculty to install Microsoft operating systems and compilers on their machines at no charge for class and research use. Students may develop in any environment they wish, as long as their code compiles and runs within Visual Studio .NET. This standardization saves the instructor and the teaching assistant significant time.

- Every student is responsible for completing their own project. Team projects are not well suited for this environment, as student-to-student differences in programming 
efficiency and ability encourage the more capable students to do the bulk of the work, making it difficult to assign individual project grades.

- To maintain accountability and a complete semester archive, each student must submit their project workspaces (source code, binary files) and data files in addition to their printed project report.

- Each student's code must be unique. While students are encouraged to discuss these projects, they are not permitted to work side by side at a computer screen or to share code electronically. After the project submission, plagiarism checking software compares every student's code against every other submission from the class.

Convolution. Learning objectives for a convolution (time-domain filtering) project are consistent from semester to semester. Upon completion of a convolution project, each student should be able to do the following:

- Calculate the unit impulse response of a system given the differential equation that describes its behavior.

- Using time-domain convolution, determine the zero-state response of a system to an arbitrary input waveform given the unit impulse response of the system.

- Utilize a circuit simulator to verify a filter's time-domain behavior

- Arrange code in a manner that promotes reuse and protects proprietary information

- Load file data, dynamically allocate memory, and work with variable length arrays

- Organize data into structures that facilitate bookkeeping and information transfer

In these projects, a student's code may load a signal from an input ASCII text file, perform a convolution between the input signal and the unit impulse response of the system (e.g., a lowpass Butterworth filter with a user-specified cutoff frequency), save the output signal to disk, and plot the original and filtered signals to the screen. Figure 2 illustrates the approach for a convolution project assigned in Spring 2003. In this project, $2^{\text {nd }}$-order Butterworth lowpass and highpass filters were cascaded to produce multiple-order lowpass, highpass, and bandpass filters. This was a semester-long project where students were required to use structures and header/source file pairs as an exercise in writing organized and maintainable code. In a normal semester, a convolution project will require less time (e.g., one month) to allow room for a frequencydomain filtering project toward the end of the semester. As implied earlier, the first project in a semester will address either convolution or Fourier series, but not both; the topics fall too closely together to allow students time to complete both efforts.

A typical graphical output for a convolution may appear as illustrated in Figure 3 . The input signal, output signal, and unit impulse response of the system are usually present. In this instance, the lower plot also depicts a subset of the individual weighted impulse responses that were summed to yield the output signal. As noted in the theory section, this approach to convolution is more intuitive than the flip-and-shift method taught in most circuits courses. Input signals for these simulations include ideal signals (e.g., pulses, square waves, sinusoids) and real-world signals (e.g., biomedical signals such as electrocardiograms and light reflectance signals from pulse oximeter sensors ${ }^{19,20}$ ). 


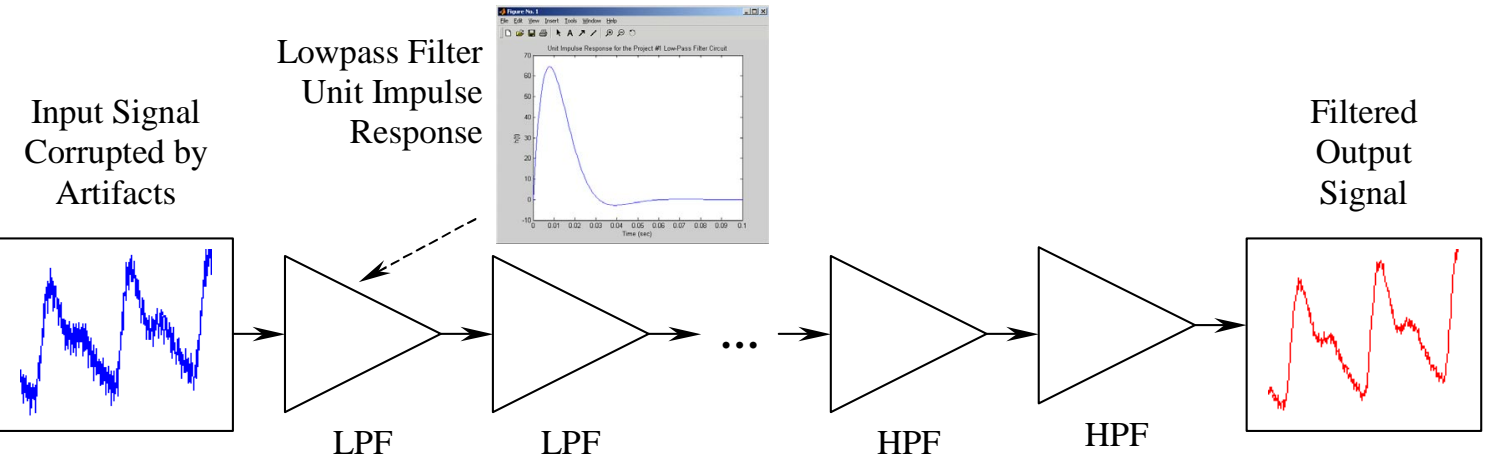

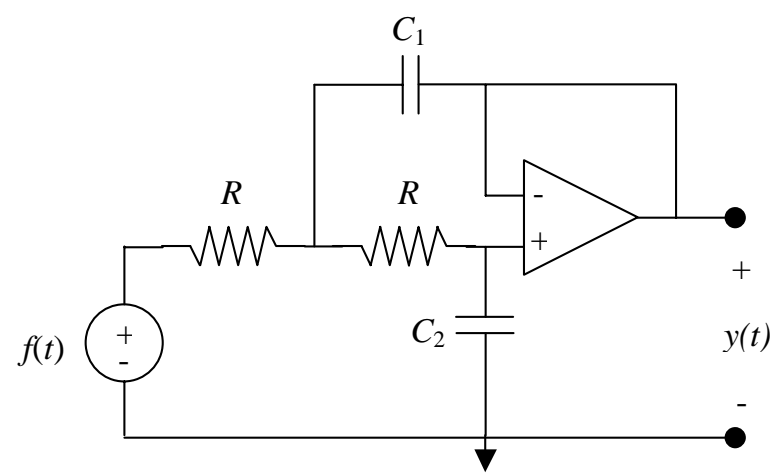

$2^{\text {nd }}$-Order Lowpass VCVS Filter

System: $\frac{d^{2} y}{d t^{2}}+\frac{2}{R C_{1}} \frac{d y}{d t}+\frac{1}{R^{2} C_{1} C_{2}} y(t)=\frac{1}{R^{2} C_{1} C_{2}} f(t)$

UIR: $h(t)=\frac{1}{R^{2} C^{2}} t e^{-t / R C} u(t)$ for $C_{1}=C_{2}$

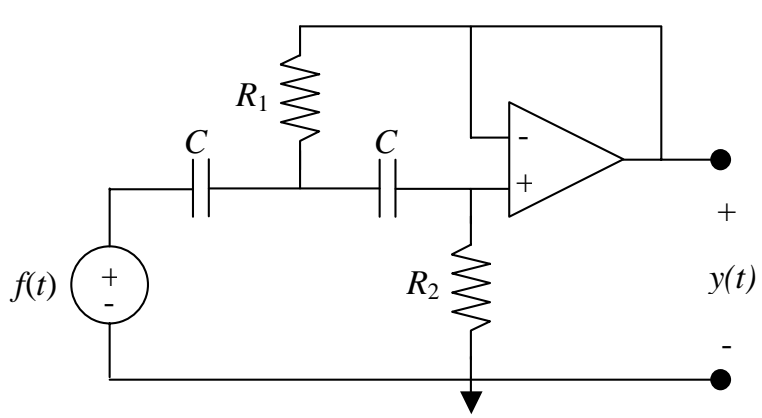

$2^{\text {nd }}$-Order Highpass VCVS Filter

System: $\frac{d^{2} y}{d t^{2}}+\frac{2}{R_{2} C} \frac{d y}{d t}+\frac{1}{R_{1} R_{2} C^{2}} y(t)=\frac{d^{2} f}{d t^{2}}$

UIR: $h(t)=\delta(t)+\left[\left(\frac{-2}{R C}+\frac{1}{R^{2} C^{2}} t\right) e^{-t / R C}\right] u(t)$ for $R_{1}=R_{2}$

Figure 2. Signal processing of light reflectance signals using cascaded filters (EECE 512). 
Input Signal: $100 \mathrm{~ms}$ Pulse

System: $2^{\text {nd }}-$ Order Lowpass VCVS Filter with $R=50 \mathrm{k} \Omega, C_{1}=0.2 \mu \mathrm{F}$, and $C_{2}=0.1 \mu \mathrm{F}$
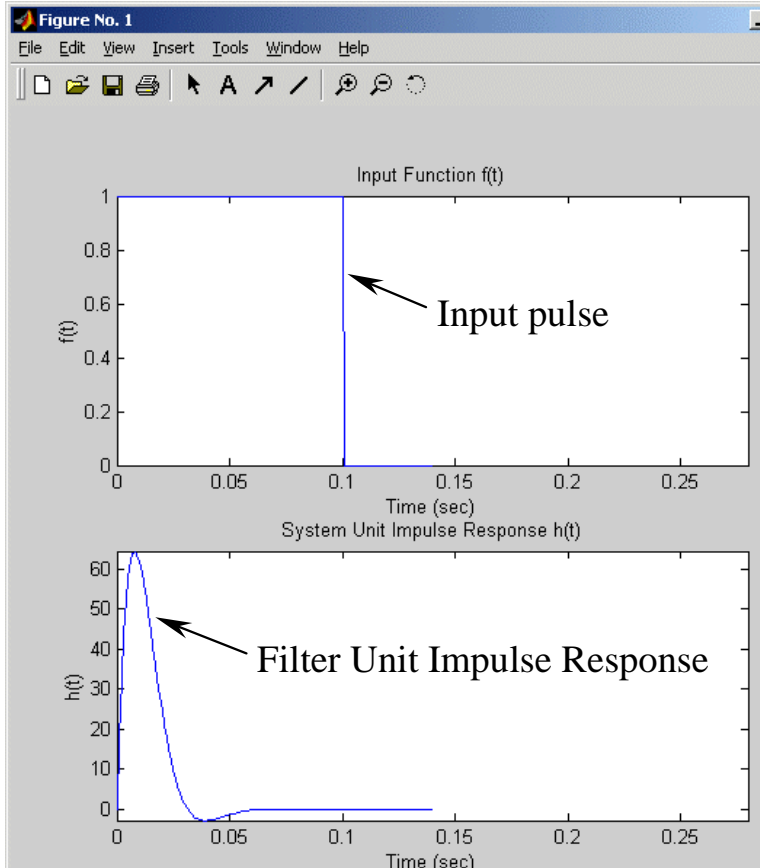

Zero-State Response as a Sum of Weighted Impulse Responses

Output Signal: Sum of the individual impulse responses

Weighted impulse responses

(not all are shown)

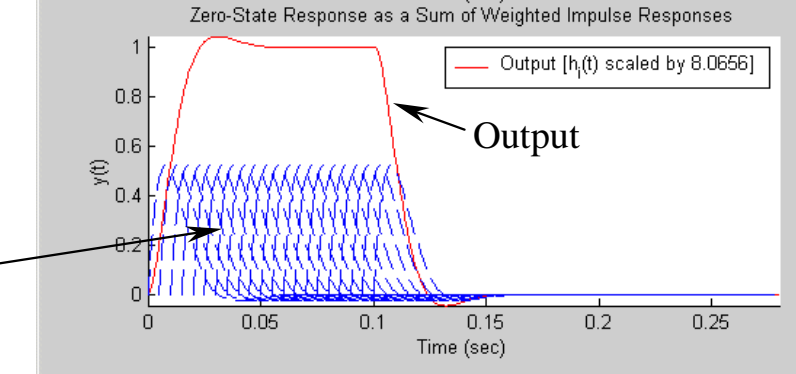

Figure 3. Example project output for a square wave convolved with the unit impulse response of a lowpass Butterworth filter.

Fourier Series. Fourier series projects support their own set of learning objectives. Upon completion of a Fourier series project, each student should be able to do the following:

- Explain how a signal can be constructed from sinusoidal building blocks

- Describe a signal in terms of its frequency domain components

- State the mathematical relationships between trigonometric, compact trigonometric, and exponential Fourier series

- Numerically calculate the Fourier series representation for a data set

- Properly sample a periodic signal so that numerically determined Fourier series coefficients are equivalent to those calculated by hand

The mechanics of a Fourier series project are similar to those of a convolution project, but the processing element involves the calculation of the Fourier series coefficients (e.g., $a_{0}, a_{n}$ and $b_{n}$ ) and the reconstruction, $f_{T F S}(t)$, of the waveform using this finite basis set. The students then develop plotting functions that allow one to visualize the reconstruction (see Figure 4). Figure 5 
illustrates the output from a Spring 2002 Fourier series project, where trigonometric Fourier series were used to reconstruct ideal and real-world signals. For this project, students also implemented calculations for both compact trigonometric and exponential Fourier series. The typical duration for these projects is about a month from start to finish.

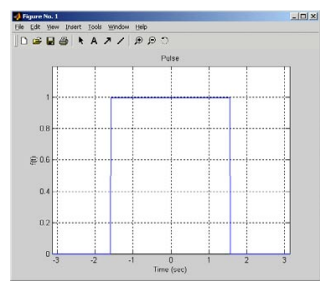

Load signal from file

$$
\begin{aligned}
a_{n}=\frac{2}{T_{0}} \int_{t_{1}}^{t_{1}+T_{0}} f(t) \cos \left(n \omega_{0} t\right) d t, \quad n=1,2,3, \ldots \\
\Longrightarrow b_{n}=\frac{2}{T_{0}} \int_{t_{1}}^{t_{1}+T_{0}} f(t) \sin \left(n \omega_{0} t\right) d t, \quad n=1,2,3, \ldots \\
f_{T F S}(t)=a_{0}+\sum_{n=1}^{\infty} a_{n} \cos \left(n \omega_{0} t\right)+b_{n} \sin \left(n \omega_{0} t\right), \quad t_{1} \leq t \leq t_{1}+T_{0}
\end{aligned}
$$

Calculate Fourier coefficients and reconstruct the signal

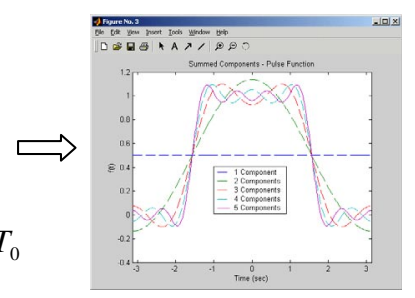

Display the results

Figure 4. Fourier coefficient calculation and signal reconstruction.

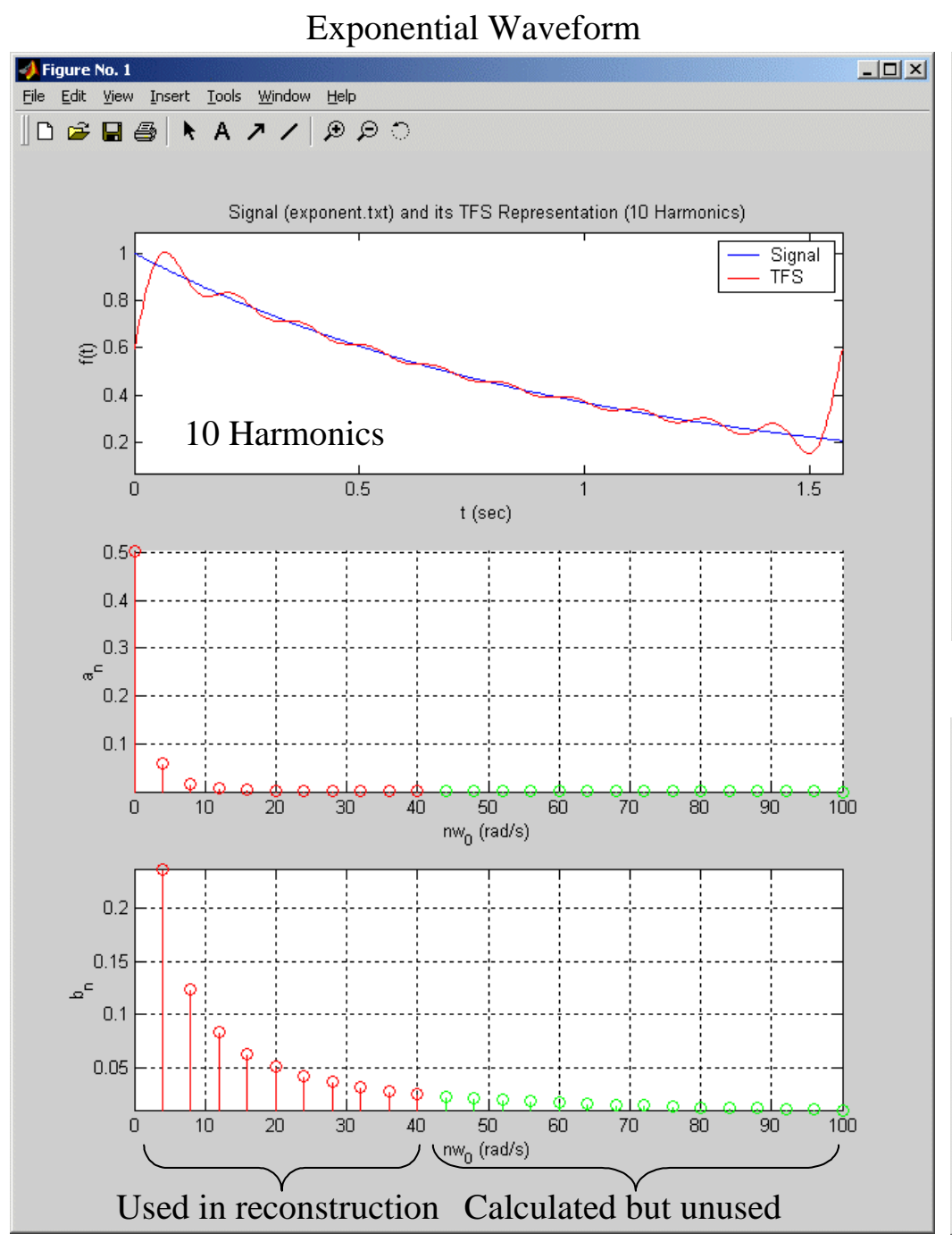

Human Pulse Plethysmogram

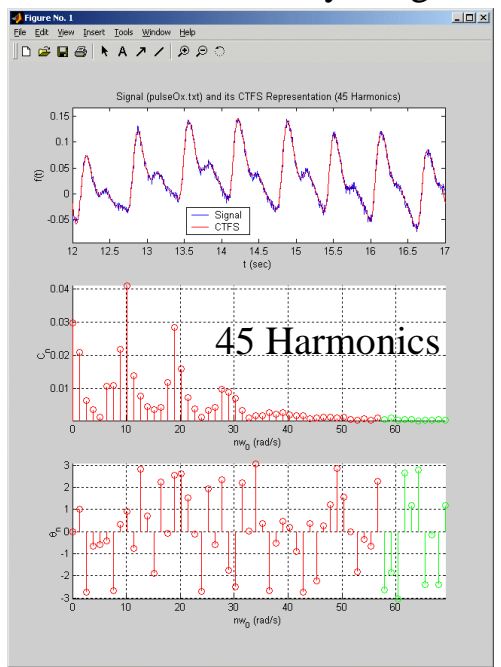

Canine Electrocardiogram

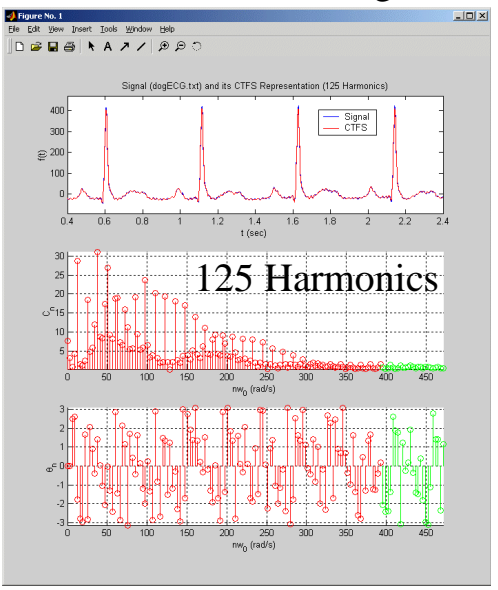

Figure 5. An exponential signal reconstruction from a Spring 2002 trigonometric Fourier series project.

Proceedings of the 2005 American Society for Engineering Education Annual Conference and Exposition Copyright @2005, American Society for Engineering Education 
Fourier Transforms and Applications. Regarding learning objectives, each student should be able to do the following upon completion of a Fourier transform project:

- Describe a signal in terms of its frequency domain components

- Construct ideal filters based upon a set of design criteria

- Perform filtering via linear convolution in the frequency domain

- Properly sample a signal to retain its important spectral information (i.e., avoid aliasing)

- Scale coefficients so that they can be compared to those calculated by hand

- Describe the effects of zero padding on signal spectra

- Create script files for GNUPLOT or MATLAB (C projects only)

The processing operations for these projects are illustrated in Figure 6. Each student's program 1. loads a signal $\left(t_{k}, f_{k}\right)$ from a file and calculates its Fourier transform, (freq $\left.r,\left|F_{r}\right|, \angle F r\right)$,

2. calculates the frequency response of a filter, (freq $r,\left|H_{r}\right|, \angle H r$ ), chosen by the user, and 3. performs a linear convolution between the two sequences $\left(y_{k}=f_{k} * h_{k}=\operatorname{IFFT}\left(F_{r} H_{r}\right)\right)$. The program then saves the input/output signals, their spectra, and the filter spectra to a set of ASCII text files and creates a plotting script that can be called by MATLAB or GNUPLOT.

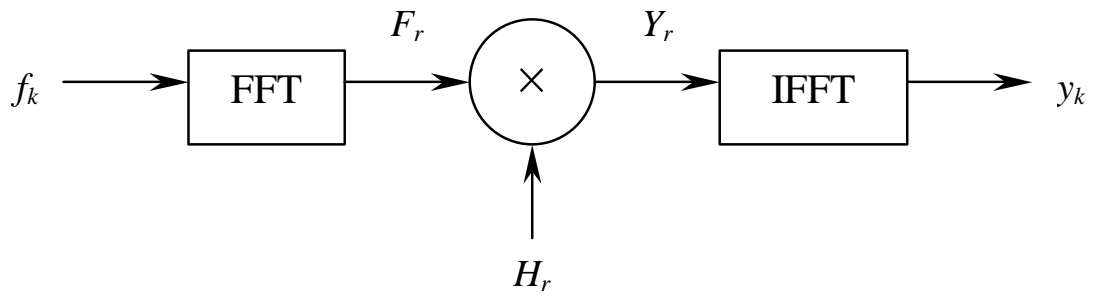

Figure 6. Linear convolution of an input sequence $f_{k}$ with a frequency domain filter $\boldsymbol{H}_{\boldsymbol{r}}$.

These filtering programs are generally applied to signals that exhibit undesirable drift and/or noise. Real-world applications heighten the interest of the students. For example, Figure 7 illustrates the screen output from a Spring 2004 project, where students used ideal frequencydomain filters to remove drift and $60 \mathrm{~Hz}$ noise from a finger photoplethysmogram obtained with a reflectance-mode pulse oximeter. ${ }^{21}$ Biomedical signals from other experiments (e.g., noisy electrocardiogram signals from kinesiology experiments) are also popular with students. In a Spring 2002 project, students digitized their own voice recordings and filtered them using a flexible frequency-domain filter such as one might employ for a tone control knob on a car stereo. $^{22}$ In other projects, students were introduced to time-frequency spectrogram tools that allow one to track spectral character versus time. Figure 8 displays time frequency spectrograms for a bat chirp and the phrase "Do you know ... the muffin man?" from the recent movie Shrek. 

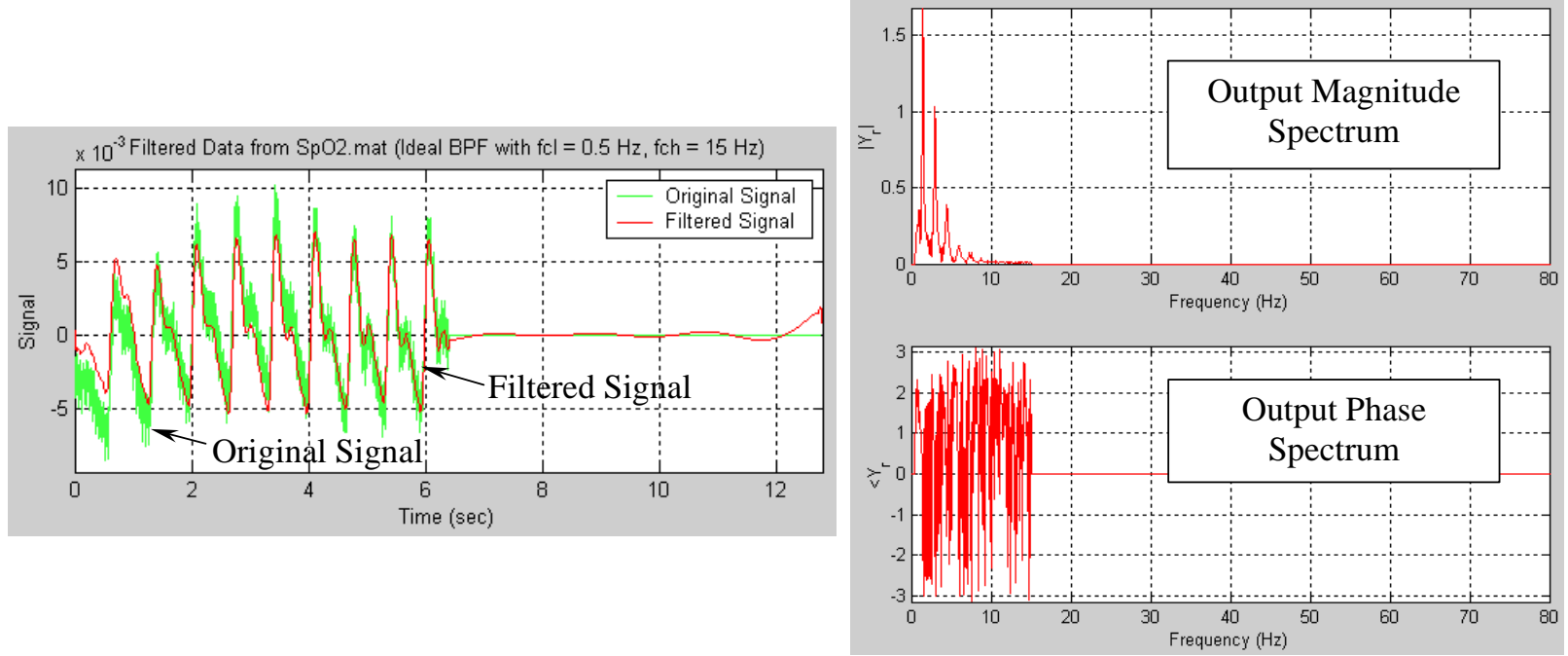

Figure 7. Representative output from a Spring 2004 frequency-domain filtering project.
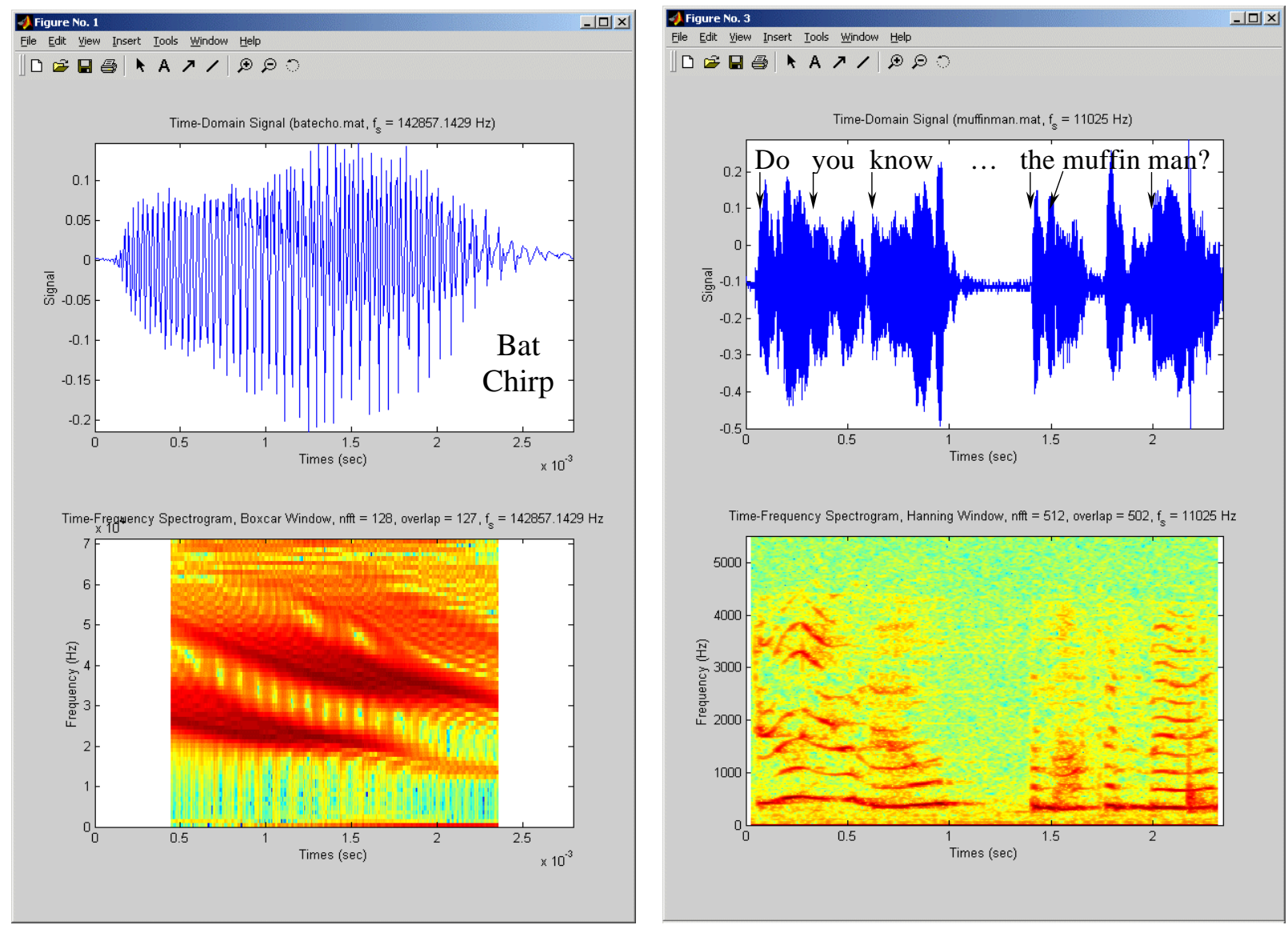

Figure 8. Screens from a Fall 2002 time-frequency project. The upper axes contain timedomain signals, while the lower axes depict the respective time-frequency distributions.

Proceedings of the 2005 American Society for Engineering Education Annual Conference and Exposition Copyright $\odot 2005$, American Society for Engineering Education 


\section{Results: Impact of Numerical Projects on Learning}

General Observations. Quantitative assessments of student learning from these projects (i.e., formal correlations between project scores and performance on individual exam problems or overall course grades) have been difficult to interpret. No clear increases or decreases in academic performance have been observed because of the subject matter addressed in the projects, likely because the project focus and programming language both change from semester to semester, and only 5 semesters worth of projects have been assessed. One can see, for example, slight increases in scores for physical convolution exam problems for the semesters this technique was utilized, but these results are not conclusive. Additionally, the format and pace of each semester are dynamic depending upon the type of project and the student population. Student learning in Linear Systems is not simply a result of how much mathematical knowledge students retain or how well they program: it also depends on the interpersonal dynamics between the students and teacher, as well as the learning environment that these foster.

However, some general observations can be made based on recent experience:

1. Good students will generally perform well, whatever it takes; poor students can perform well if properly cared for and motivated.

2. Procrastination is the largest issue that students face with these large projects. Were they better able to manage their time, students would learn much more and have a more positive project experience (see the next section).

3. Students have a love/hate relationship with project-oriented courses. They love the idea of a project, the satisfaction from getting it to work, and designing for real-world applications (see the next section). However, they dislike the growing pains associated with trying to manage a programming learning curve and project design elements during a busy semester.

4. Accountability on behalf of the student and teacher is important: students must be held accountable for project deadlines and plagiarism (a constant issue in this age of connectivity), while instructors must state clear learning objectives and offer the help that they promise.

5. Multi-week projects, while harder to organize and manage, are more satisfying to students and offer a more realistic experience. Staged deadlines help students to manage their time.

6. Students prefer MATLAB over $\mathrm{C}$ for a course of this nature.

7. Proper scoring rubrics can make the assessment experience more palatable for instructors. Observations from the perspectives of the students are addressed in the next section.

\section{Comments from Students.}

In Spring 2003 (see Figure 2), the author presented the students with a survey whose goal was to assess, from the students' perspectives, the Linear Systems semester project in terms of its viability as a learning tool. While surveys are not presented here (and were not given) for every semester that projects were assigned, this semester provides a good 'worst case' scenario: of the projects assigned to date, it was not only the most extensive in scope and duration, but it also required more from the students in terms of source code design.

The first part of the survey addressed the project learning objectives: tasks that a student should be able to perform upon completion of the project. The survey asked them, on a scale of 1 to 5 , 
to note their level of comfort with the learning objectives, where " 1 " implies no comfort and "5" implies high confidence. The average results from 49 responses are tabulated in Table 1, where the last column indicates the apparent improvement in comfort level for each of the subject areas. Note that only one survey was given, so the first column of numbers represents what students recalled from when they were assigned the project. It is sensible and encouraging to note that the greatest perceived improvements came in topic areas that the students learned during the Linear Systems course: they had used circuit simulators and worked with source code in prior semesters. For each objective, the students had an overall comfort level of 3.5 to 3.8, which indicates that the learning experience was effective, although these thoughts should be supplemented by the students' thoughts regarding the overall project, which are presented below.

Table 1. Student survey responses regarding the learning objectives of the project.

\begin{tabular}{|l|c|c|c|}
\hline Learning Objective & $\begin{array}{c}\text { Prior to } \\
\text { the Project } \\
\text { (Average) }\end{array}$ & $\begin{array}{c}\text { After the } \\
\text { Project } \\
\text { (Average) }\end{array}$ & $\begin{array}{c}\text { Change in } \\
\text { Value }\end{array}$ \\
\hline $\begin{array}{l}\text { Utilize a circuit simulator to verify a filter's } \\
\text { transfer function and transient response }\end{array}$ & 2.7 & 3.8 & +1.2 \\
\hline Implement filtering in the time domain & 1.8 & 3.8 & +2.0 \\
\hline $\begin{array}{l}\text { Determine the frequency content of a previously } \\
\text { uncharacterized signal }\end{array}$ & 1.6 & 3.6 & +2.0 \\
\hline $\begin{array}{l}\text { Design filter cascades that balance the tradeoffs } \\
\text { between artifact removal and the retention of } \\
\text { important signal information }\end{array}$ & 1.6 & 3.5 & +2.0 \\
\hline $\begin{array}{l}\text { Arrange source code in a manner that promotes } \\
\text { reuse and protects proprietary information }\end{array}$ & 2.5 & 3.8 & +1.3 \\
\hline $\begin{array}{l}\text { Organize data into structures that facilitate } \\
\text { bookkeeping and information transfer }\end{array}$ & 2.3 & 3.7 & +1.4 \\
\hline
\end{tabular}

As noted earlier, Linear Systems was chosen as a course where students would be exposed to C programming at least one more time prior to graduation. The survey asked the students, on a scale of 1 to 5, to note their level of proficiency in the given programming areas, where a " 1 " denotes no proficiency and a " 5 " denotes a solid understanding of the concept. Table 2 lists the student responses to these elements, where it is clear that the overall improvements (again, as perceived by the students) were less dramatic that those presented in Table 1. It is sensible that the larger relative changes occurred in the areas of dynamic memory allocation, structures, and function parameters. These areas are introduced in prerequisite programming courses but not given as much emphasis because they are introduced late in the semester. 
Table 2. Student survey responses regarding $\mathrm{C}$ programming elements of the project.

\begin{tabular}{|l|c|c|c|}
\hline C Programming Area & $\begin{array}{c}\text { Prior to } \\
\text { the Project } \\
\text { (Average) }\end{array}$ & $\begin{array}{c}\text { After the } \\
\text { Project } \\
\text { (Average) }\end{array}$ & $\begin{array}{c}\text { Change } \\
\text { in Value }\end{array}$ \\
\hline Standard input/output & 3.1 & 4.2 & +1.1 \\
\hline File input/output & 2.9 & 4.2 & +1.2 \\
\hline Dynamic memory allocation & 2.4 & 4.0 & +1.6 \\
\hline Structures & 2.1 & 3.8 & +1.6 \\
\hline Structures/arrays as function parameters & 2.2 & 3.7 & +1.5 \\
\hline Calculation of expressions & 3.6 & 4.3 & +0.7 \\
\hline Function prototypes & 2.8 & 3.8 & +1.1 \\
\hline Header/source file pairs & 2.7 & 4.0 & +1.3 \\
\hline Conceptual difference between compiling and linking & 2.7 & 3.5 & +0.8 \\
\hline
\end{tabular}

To understand the students' general perceptions of the overall project, the survey included the items listed in Table 3. It is encouraging to note that these students were able to get most of their code working. While a higher level of interest in the material would be desirable, this is a required course that contains both mathematical and programming elements. Nonetheless, the students clearly put an effort into this project, which extended over most of the semester, and they found the staged deadlines to be helpful. The last row in Table 3 is intriguing, because students have access to many computational workstations in the KSU EECE Department and can install the compilers at home. ${ }^{18}$ One reason for this response may be that many of the students would start each portion of the project late, so the available computer stations would often be filled the day or two before a project update was due.

Table 3. Student survey responses regarding the overall project elements.

\begin{tabular}{|l|c|}
\hline Item & Response \\
\hline Percentage of the code you were able to get working $(1=20 \%, 2=40 \%$, etc. $)$ & 4.2 \\
\hline Level of interest in the material $(1=$ none, $5=$ very interested $)$ & 3.4 \\
\hline Level of effort required (1 = too little; $5=$ too much) & 4.0 \\
\hline Staged deadlines (1= no help; 5 = very effective) & 4.1 \\
\hline Computer and software resources (1 = inadequate; 5 = adequate) & 3.7 \\
\hline
\end{tabular}

Finally, students were asked open-ended questions regarding what they liked the most (and the least) about this project. These thoughts are summarized in Table 4 . Students clearly received satisfaction from getting their code to work and enjoyed working with real-world data. However, they struggled with the programming elements, which is natural for any programming language, especially given the time management constraints of a busy semester course schedule. 
Table 4. What students liked the most (and the least) about this project.

\begin{tabular}{|l|c|}
\hline What Students Liked the Most & $\begin{array}{c}\text { Number of } \\
\text { Responses from } \\
\text { 49 Surveys }\end{array}$ \\
\hline Actually implementing a filter and getting it to work & 15 \\
\hline Signal processing on real-world signals & 11 \\
\hline Working with filters and signal processing & 9 \\
\hline Staged project deadlines & 5 \\
\hline & \\
\hline What Students Liked the Least & 21 \\
\hline C programming & 5 \\
\hline Uncertainty regarding how the output signal should look & 4 \\
\hline Memory allocation & 4 \\
\hline Debugging C code & \\
\hline
\end{tabular}

\section{Conclusions}

This paper presents experiences with multi-week, numerical computation projects that address time- and frequency-domain concepts in upper-level undergraduate courses. Project descriptions and examples of the graphical plots produced by these programs are provided. From these experiences, it is first clear that good students will generally perform well on projects, whatever it takes, because they have consistent expectations for their academic performance. Poor students can perform well, but caring and motivation must be received from the instructor.

Second, students appreciate the idea of a large project, the satisfaction from getting it to work, and the ability to design around real-world applications. However, they also experience the discomfort that naturally arises from managing project schedules and learning curves.

Procrastination is the largest issue that students face in these large projects. Students state that staged deadlines for these multi-week efforts help to alleviate this issue.

Third, accountability on behalf of both student and teacher is important: students must be held accountable for project deadlines and plagiarism, while instructors must state clear learning objectives and offer the resources necessary for the student to complete the effort.

While learning is difficult to quantify in this environment, past experience and feedback from students indicates that these multi-week projects drive home concepts such as convolution, fast Fourier transforms, and frequency-domain filtering that are difficult to absorb from lecture notes and short-term, episodic handwritten exercises.

\section{Acknowledgements}

Portions of this material are based upon work supported by the National Science Foundation under grant BES-0093916. Opinions, findings, conclusions, or recommendations expressed in this material are those of the author(s) and do not necessarily reflect the views of the NSF.

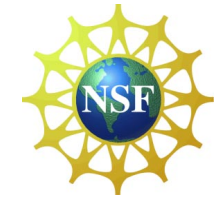




\section{References}

[1] Bloom, Benjamin S., Bertram B. Mesia, and David R. Krathwohl. Taxonomy of Educational Objectives (Two Volumes: The Affective Domain \& The Cognitive Domain): New York, David McKay, 1964.

[2] Bloom, Benjamin S. and David R. Krathwohl. Taxonomy of Educational Objectives: The Classification of Educational Goals, by a committee of college and university examiners. Handbook I: Cognitive Domain: New York, Longmans, Green, 1956.

[3] Greenberg, Julie E., Bertrand Delgutte, and Martha L. Gray. "Hands-On Learning in Biomedical Signal Processing," IEEE Engineering in Medicine and Biology Magazine, July/August, pp. 71-79.

[4] Taylor, Fred, Jonathan D. Mellott, and Michael Lewis. "Spectra - A Hands-on DSP Learning Experience," presented at 1996 IEEE International Conference on Acoustics, Speech, and Signal Processing, 1996.

[5] Butler, Frank, Rebecca Whitehead, and Mark Winkleman. "Student, Alumni and Employer Satisfaction to a Collaborative Learning Approach," 31 ${ }^{\text {st }}$ Annual Frontiers in Education Conference, Reno, NV, 2001.

[6] Azemi, A. and E.E. Yaz. "Using Graphical User Interface Capabilities of MATLAB in Advanced Electrical Engineering Courses," Proceedings of the $38^{\text {th }}$ IEEE Conference on Decision and Control, 1999.

[7] Hill, Andrew, Flora McMartin, and Alice Agogino. "Creating Online Faculty Collaboration to Develop Engineering Education Computer Learning Materials," presented at 31st Annual Frontiers in Education Conference, Reno, NV, 2001.

[8] Bonham, Scott and Robert Beichner. "Online homework: Does It Make a Difference?," http://physics.wku.edu/ bonham/Publications/HomeworkCompare.pdf.

[9] Lathi, B.P. Signal Processing \& Linear Systems, First ed: Berkeley-Cambridge Press, Carmichael, CA, 0195219-17-1, 1998.

[10] Veen, Simon Haykin and Barry Van. Signals and Systems, Second ed. New York: John Wiley and Sons, 0471-16474-7, 2003.

[11] Chen, Chi-Tsong. Signals and Systems, $3^{\text {rd }}$ Ed., New York: Oxford University Press, 0-19-515661-7, 2004.

[12] Sherrick, John D. Concepts in Signals and Systems, Second ed. Upper Saddle River, NJ: Prentice Hall, 013-178271-1, 2005.

[13] Delores M. Etter, David C. Kuncicky, and Holly Moore. Introduction to MATLAB 7, First ed. Upper Saddle River, NJ: Prentice Hall, 0-13-147492-8, 2005.

[14] Littlefield, Duane Hanselman and Bruce. Mastering MATLAB 7, First ed. Upper Saddle River, NJ: Prentice Hall, 0-13-143018-1, 2005.

[15] Heath, Michael T. Scientific Computing: An Introductory Survey, Second ed. New York, NY: McGrawHill, 0-07-239910-4, 2002.

[16] Christensen, Mikael. "Syntopia," 2002, http://syntopia.sourceforge.net/.

[17] Cadence Design Systems, Inc. "OrCAD," 2005, http://www.orcadpcb.com/.

[18] Corporation, Microsoft. "Microsoft Academic Alliance Developer Center," 2005, http://msdn.microsoft.com/academic/.

[19] Warren, Steve, Jianchu Yao, Ryan Schmitz, and Luke Nagl. "Wearable Telemonitoring Systems Designed with Interoperability in Mind," $25^{\text {th }}$ Annual Conference of the IEEE EMBS, Cancun, Quintana Roo, Mexico, 2003.

[20] Yao, J., and S. Warren. "Design of a Plug-and-Play Pulse Oximeter," $2^{\text {nd }}$ Joint EMBS-BMES Conference, Houston, TX, 2002.

[21] Love, Jerry T., S. Warren, G. R. Laguna, and T. J. Miller. " Personal Status Monitor," Sandia National Laboratories, SAND97-0418, 1997.

[22] Marttila, Ilkka. "Second-Order Audio Filter Performs Multiple Functions," Electronic Design, April 30, 2001, pp. 91-92.

\section{Biographical Information}

Steve Warren is an Associate Professor of Electrical \& Computer Engineering at Kansas State University. He teaches courses in linear systems, computer graphics, biomedical instrumentation, and scientific computing. Dr. Warren manages the KSU Medical Component Design Laboratory, and his research targets plug-and-play, wearable systems for telemedicine. 